\title{
Warm extremes: Marine Isotope Stage 5e and its relevance for the future
}

\author{
Eleonora Regattieri' ${ }^{1}$ P. Scussolini ${ }^{2}$, W.-L. Chan ${ }^{3}$ and S. Sherriff-Tadano ${ }^{3}$
}

\section{QUIGS-PMIP workshop, Cambridge, UK, 1-4 July 2019}

\section{The QUIGS working group}

(pastglobalchanges.org/quigs) aims to understand climate and environmental changes during quaternary interglacials, by integrating paleoclimatic records and climate model simulations.

This workshop (pastglobalchanges.org/ calendar/2019/127-pages/1910) kicked off QUIGS' second phase (2019-2021), where improved datasets and new model experiments are being used to address research questions and knowledge gaps identified during QUIGS' first phase (2015-2017). The meeting focused mainly on the Last Interglacial (LIG, known in the marine record as Marine Isotope Stage (MIS) 5e) and its relevance for understanding Earth-system responses to ongoing and future climate change, although other past warm periods were also considered.

A total of 40 delegates from 12 countries participated. The first day was dedicated to discussing the latest results of the new PMIP4 climate simulations. Experiments on the LIG and mid Holocene ( $\mathrm{MH}$ ) based on the Tier 1 PMIP4 protocol are complete, ongoing, or planned for most models, and include transient simulations and experiments with different ice sheets, vegetation, and freshwater fluxes. Results will be analyzed by adopting the fixed-angular celestial calendar, essential to studying seasonality across geological timescales. Latest modeling results for the LIG consistently show summer warming at
Northern Hemisphere high latitudes, marked strengthening of boreal monsoons, and weakening of austral monsoons. Several modeling studies also pointed out the importance of vegetation feedback on the amplitude of Northern Hemisphere warming. Results in the Southern Hemisphere are not as consistent: e.g. the Southern Ocean warms up or cools down depending on the model, hinting at different mechanisms at work.

From the second day, the spotlight expanded to proxy-based climate reconstructions and proxy-model comparison. A series of invited talks reviewed the current knowledge of LIG climate. Improved reconstructions of global LIG surface temperature anomalies (relative to the pre-industrial) from marine, ice-core, and continental records were presented (Fig. 1), highlighting that seasonal biases of many records still have to be addressed, and giving recommendations for the comparison to simulations of the 127 kyr BP snapshot. The latest progress in high-resolution greenhouse gas reconstruc tions from EPICA Dome $\mathrm{C}$ were presented, reporting, e.g. more stable $\mathrm{CO}_{2}$ concentrations during the LIG than previously thought. Regarding LIG sea level, the latest estimates of its peak height and temporal evolution were presented, and the main sources of uncertainty were systematically identified. Challenges still to be addressed by the wider community include improved constraints on MIS 6 ice sheets, through both paleorecords

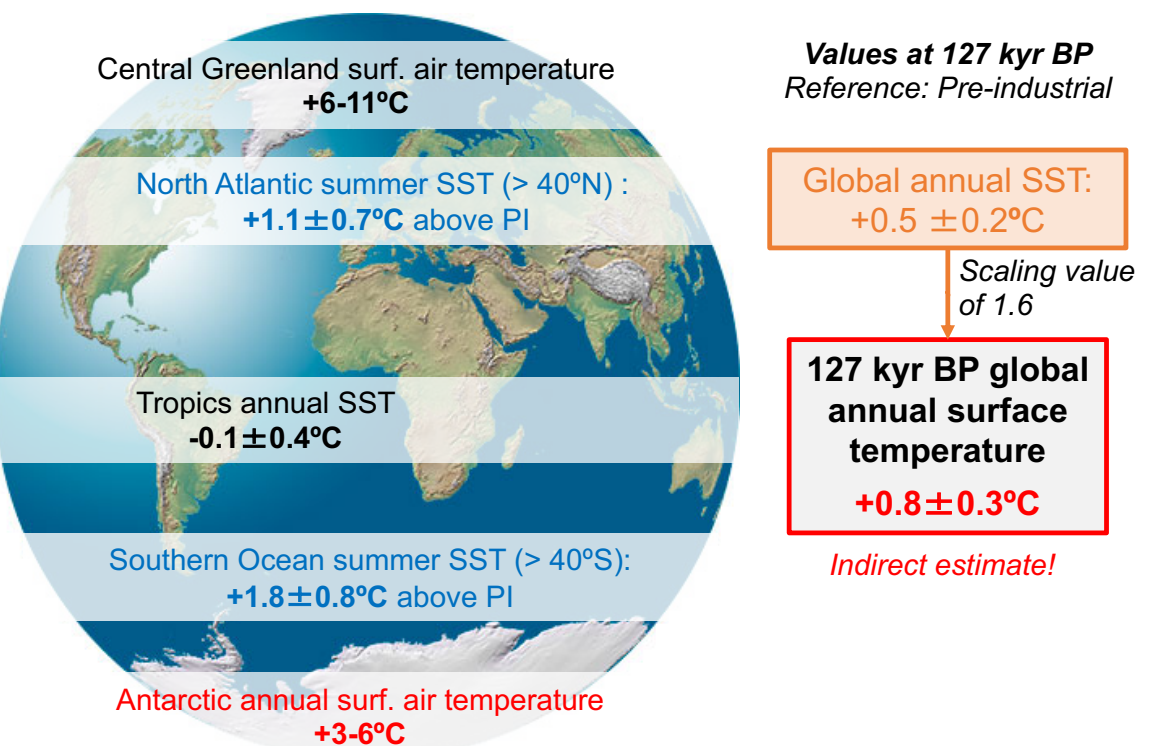

Figure 1: Summary of LIG surface temperature anomalies from marine sediment and ice-core records (courtesy of E. Capron; estimates deduced from datasets using approaches presented by Capron et al. 2017, Hoffman et al. 2017, and Fischer et al. 2018). and modeling, and a proper understanding of the magnitude of LIG melting from Greenland and Antarctica.

Another slot of invited talks summarized the latest advances in LIG and $\mathrm{MH}$ modeling. The LIG precipitation from the new PMIP4 runs compares favorably to new proxy-based global synthesis. Some modeling groups focus on monsoon regions and on high boreal latitudes.

The meeting also saw a series of selected proxy talks and posters considering different types of globally distributed archives and proxies. Topics ranged from estimates of mean ocean temperature, polar sea-ice extent, and regional sea-level variations, to syntheses of micropaleontological and pollen records.

Discussions in plenum and in working groups identified the main challenges still to be addressed by both communities and suggested possible ways forward. These issues related to (i) chronological mismatching and seasonal biases in climate reconstructions: (ii) uncertainties in the MIS 6 ice-sheet extent and distribution, essential to an accurate modeling of LIG climate and sea level; (iii) uncertainties in vegetation evolution during the LIG interval, also important for numerical simulations; and (iv) uncertainties in sea ice, important for understanding the dynamics of Arctic amplification.

A short-term key goal was established for the QUIGS community to strive to publish relevant LIG science in time to contribute to the forthcoming 6th Assessment Report of the IPCC (AR6). In the longer term, focus will be placed on unraveling specific mechanisms, such as interactions between ice sheets, ocean, climate, and vegetation, and on comparing simulations of the LIG to those of the MH, and PMIP4 to PMIP3 and other CMIP simulations.

\section{AFFILIATIONS}

${ }^{1}$ University of Pisa, Italy

'Vrije Universiteit Amsterdam, Netherlands

3University of Tokyo, Japan

\section{CONTACT}

Eleonora Regattieri: eleonora.regattieri@unipi.it

REFERENCES

Capron E et al. 2017 Quat Sci Rev 168: 137-150

Hoffman JS et al. 2017 Science 355: 276-279

Fischer Het al. 2018 Nat Geosci 11: 474 